\title{
Medicus amicus. Un trait romain dans la médecine antique
}

Par Philippe Mudry

Dans la préface qui ouvre la première partie, consacrée à la diététique, de son traité De la médecine ${ }^{1}$, l'encyclopédiste latin Celse expose successivement les doctrines antagonistes des écoles médicales dogmatique et empirique, avant d'exprimer, sous la forme d'une vaste réflexion méthodologique, son opinion personnelle sur le différend qui oppose ces deux écoles. Partisan de la prise en compte par le médecin des caractères individuels de l'affection, Celse y réfute longuement et vivement la doctrine, nouvelle à son époque, de l'école méthodique qui, dans la détermination de la thérapeutique, ne veut retenir que les caractères communs (resserrement - relâchement). Cette réfutation dans laquelle Celse affirme, exemples à l'appui, la nécessité de l'individualisation du malade, se termine sur une phrase qui apparaît comme la conclusion de toute la démonstration qui précède: «c'est pourquoi, à science égale, le médecin est plus efficace s'il est un ami que s'il est un étranger». ${ }^{2}$

Ce jugement de Celse exprime une conception de la relation entre le médecin et le malade qui nous paraît inconnue de la médecine grecque. En effet, qu'un lien d'amitié entre le médecin et le malade assure une thérapeutique plus efficace est une idée qui, à notre connaissance, ne se trouve nulle part dans la littérature médicale grecque, de la Collection hippocratique à Galien. C'est qu'une telle relation ne doit pas être confondue avec la fameuse philanthropie hippocratique, ce sentiment d'humanité et de compassion qui, selon Hippocrate, doit animer le médecin et l'inciter à soulager les souffrances d'autrui. Cette conception hippocratique du métier de médecin, dans laquelle l'amour de l'art est inséparable de l'amour de l'humanitée ${ }^{3}$, a façonné la déontologie médicale des siècles suivants. Elle se retrouve particulièrement vive dans la réaction des médecins empiriques contre ce qu'on pourrait appeler «l'hypertechnicité» de la médecine dogmatique préoccupée davantage, selon les empiriques, de la maladie que du malade, et dans leur volonté de mettre l'individu à guérir au centre des préoccupations du médecin. C'est d'ailleurs au nom de ces sentiments d'humanité et de l'idée qu'ils se font de leur profession, conçue comme un art au service de la vie ${ }^{4}$, que les médecins empiriques se sont élevés avec tant de véhémence, à ce que rapporte Celse ${ }^{5}$, contre la vivisection humaine, telle qu'elle aurait été pratiquée à Alexandrie: parce que, disaient-ils, le médecin peut acquérir les connaissances anatomiques nécessaires à l'exercice de son art en soignant les blessés plutôt qu'en assassinant d'autres hommes, fussent-ils des criminels, en faisant preuve d'humanité, 
per misericordiam, plutôt que de cruauté. Nous pourrions citer aussi Scribonius Largus, ce médecin du $1^{\text {er }}$ siècle ap. J.-C., auteur d'un traité sur les médicaments ${ }^{6}$, selon qui le médecin trahit l'esprit de sa profession s'il n'a le cœur rempli de compassion et d'humanité. Mais nous trouverions encore et toujours l'expression d'un sentiment de pitié devant la souffrance humaine et un besoin de lui venir en aide, jamais, en revanche, la mention d'une relation personnelle d'amitié entre le médecin et le malade.

Ce n'est pas le lieu de nous interroger ici sur les raisons pour lesquelles la notion d'amitié entre médecin et malade est restée inconnue tout au long de l'histoire de la médecine grecque. Il suffit à notre propos de la constater. Disons simplement, en nous référant à des travaux récents ${ }^{7}$, que, tout au moins en ce qui concerne le médecin hippocratique, une telle idée n'a pu que lui être étrangère et même, en un certain sens, contraire. En effet, le médecin hippocratique est un itinérant, un périodeute, qui, par la force des choses, ne peut avoir avec son patient la longue fréquentation que suppose un lien d'amitié. Il est, en outre, dépourvu de toute reconnaissance officielle, de tout diplôme qui sanctionnerait ses connaissances. C'est la réputation qu'il se crée qui lui tient lieu de statut professionnel. Aussi est-il poussé à maintenir envers ses patients une sorte de distance objective qui lui permet de faire impression, par exemple, en devinant le passé morbide d'un malade à partir du seul examen clinique et parfois même, comme on le voit dans le premier chapitre du Pronostic, sans poser de question.

Le jugement de Celse qui considère la relation d'amitié entre le médecin et son malade comme le moyen d'une plus grande efficacité thérapeutique, se présente, ainsi que nous l'avons signalé plus haut, comme l'aboutissement de la longue réfutation du méthodisme que Celse vient de développer. Il ne s'agit donc pas d'une remarque que Celse ferait en passant, mais d'un aspect essentiel de la méthodologie médicale qui est la sienne. Considérant que la doctrine méthodique des caractères communs est rudimentaire, tout juste bonne pour les vétérinaires ou les médecins des grands hôpitaux, les ualetudinaria, qui sont confrontés à des foules de malades, Celse ${ }^{8}$ fonde sa réfutation sur l'impérieuse nécessité qu'il y a à prendre en compte l'individualité du malade et l'ensemble des caractères particuliers que constituent le tempérament, la constitution, l'âge, les antécédents morbides, le mode de vie etc... Les éléments de cette démonstration s'inscrivent tous dans la tradition médicale grecque. L'observation des caractères particuliers du malade est, en effet, un des traits dominants de la médecine grecque et les arguments de Celse en faveur d'une médecine individualisée sont exactement ceux que dogmatiques et empiriques utilisent régulièrement pour combattre le métho$\operatorname{disme}^{9}$. Mais cette individualisation objective et «scientifique» ${ }^{10}$ se prolonge 
chez Celse par une individualisation subjective car, il est essentiel de le noter, Celse se réfère dans sa comparaison à deux médecins dont le niveau de connaissances est semblable, cum par scientia sit ${ }^{11}$. La différence d'efficacité entre l'un et l'autre médecin se situe donc ailleurs que sur le terrain de la science médicale: il existe pour Celse une connaissance médicale qui ne se mesure pas en termes de science, une sorte de connaissance de l'intérieur, de sensibilité à la nature profonde du malade, qui constitue, sur un autre plan, la prolongation de l'individualisation objective du malade et qui passe par une relation d'amitié avec le malade.

En introduisant dans la médecine qu'il préconise la notion de medicus amicus, Celse s'inspire, croyons-nous, de l'antique tradition médicale de Rome telle que, au témoignage de Pline $^{12}$, elle se maintint pendant plus de six siècles avant d'être submergée, à partir du $2^{\mathrm{e}}$ siècle av. J.-C., par l'irruption de la médecine grecque et son succès rapide. Celse est un nostalgique du passé, comme en témoigne, au début du De medicina ${ }^{13}$, la longue digression dans laquelle il considère que la complexité de la médecine contemporaine est en relation directe avec le relâchement des mœurs, et qu'autrefois une telle médecine n'était pas nécessaire, car les mœurs meilleures garantissaient aussi une santé meilleure. Une telle attitude d'esprit ne signifie pourtant pas que Celse songe à revenir à la médecine du chou et aux pratiques rudimentaires de Caton. Son traité entièrement fondé sur la médecine grecque de même que l'absence, dans l'historique qu'il trace de la médecine ${ }^{14}$, de toute référence à la médecine romaine d'autrefois en sont bien la preuve. Ce que Celse tente de retrouver n'est pas la médecine romaine d'autrefois, mais le médecin romain d'autrefois, ou plus exactement le type de relation qui existait autrefois entre celui qui remplissait la fonction de médecin, c. à d. le chef de famille, le paterfamilias, et le malade. Il est significatif, à ce point de vue, que Celse oppose le médecin ami et le médecin extraneus ${ }^{15}$, c. à d. celui qui vient du dehors, qui est étranger dans la maison où on l'appelle. La volonté de Celse d'introduire, par le biais du medicus amicus, un trait romain dans la médecine contemporaine qui, à Rome, est grecque aussi bien par son contenu doctrinal que par ses représentants, s'explique d'autant mieux que l'école méthodique rencontre alors un succès grandissant. Or rien n'est plus contraire à la médecine familiale d'autrefois que cette médecine nouvelle qui, à ce qu'en rapporte Celse, néglige l'individu et soigne de façon impersonnelle comme on le fait avec des animaux. En concluant sa réfutation du méthodisme sur la supériorité thérapeutique du medicus amicus, Celse propose, contre cette doctrine, un modèle de praticien qui est grec dans ses connaissances professionnelles et romain dans sa relation au malade. Un médecin qui, mutatis mutandis, représenterait assez bien ce que nous appelons aujourd'hui, avec quelque nostalgie, le médecin de famille. 
${ }^{1}$ Corpus Medicorum Latinorum I, 1915, Marx.

${ }^{2}$ I préf. 73: Ideoque, cum par scientia sit, utiliorem tamen medicum esse amicum quam extraneum.

${ }^{3}$ Hippocrate, Préceptes $6(=9,258 \mathrm{~L})$.

${ }^{4}$ Celse I préf. 40 : salutis humanae praesidem artem.

${ }^{5}$ I préf. 40 sqq.

${ }^{6}$ Compositiones (Helmreich, 1887).

${ }^{7}$ Ch. Lichtenthaeler, $8^{e}$ étude hippocratique, De l'économie du Pronostic d'Hippocrate, in: $4^{\mathrm{e}}$ série d'études hippocratiques, pp. 50 sqq. (Genève, 1963).

H.Koelbing, Arzt und Patient in der antiken Welt, pp. 80 sqq. (Zürich/Stuttgart, 1977). ${ }^{8}$ I préf. 65 sqq.

${ }^{9}$ cf. Galien, De sectis ad eos qui introducuntur, Scripta minora 3, pp.18 sqq. (1893, Helmreich).

10 Nous mettons ce terme entre guillemets car, dans le domaine de l'Antiquité, il ne peut être entendu dans le sens rigoureux qui est le sien aujourd'hui. Cf. R. Joly, Le niveau de la science hippocratique, pp.15 sqq. (Paris, 1966).

11 voir supra note 2 .

12 nat. $29,11$.

${ }^{13}$ I préf. 4 sq.

${ }^{14}$ I préf. 1-11.

${ }^{15}$ voir supra note 2 .

\section{Summary}

The author discusses the relation between the physician and his patient in the antiquity, especially the question, if personal friendship between both is favorable for the cure, or if this is not the case.

Philippe Mudry

Rouvraie 8

CH-1018 Lausanne 